\title{
Evaluation of the Inhibitory Potential of Beta Lactam Derivatives Against Bile Salt Hydrolase from Enterococcus Faecalis by In-Silico Approach
}

\author{
Neha Sharma M ${ }^{1}$, Smiline Girija A.S² and J.Vijayashree Priyadharsini J ${ }^{4}$ \\ ${ }^{1}$ Saveetha Dental College and Hospitals, Saveetha Institute of Medical \\ and Technical Sciences, Saveetha University, Chennai, India \\ ${ }^{2}$ Associate Professor, Department of Microbiology, Saveetha Dental College and Hospitals, \\ Saveetha Institute of Medical and Technical Sciences, Saveetha University, Chennai, India \\ ${ }^{3}$ BRULAC-DRC, Saveetha Dental College and Hospitals, Saveetha Institute of Medical and Technical \\ Sciences [SIMATS], Saveetha University, P.H.Road, Chennai, Tamilnadu - 600077, India
}

\begin{abstract}
Bile Salt Hydrolase (BSH) is a vital protein in E.faecalis virulence and recent studies implicate that it can be an alternative target for the beta lactam derivatives to combat the menace of its complications in endodontic infections. The aim of the present study is thus undertaken to evaluate the inhibitory potential of four beta lactam derivatives against the BSH of E.faecalis. 3D structure of BSH was retrieved from the PDB data bank with further optimization of both the protein and ligands. In-silico inhibitory potential of the selected beta lactam derivatives against BSH was done by AutoDock 2.0 and was visualized with accelrys studio discoverer with the assessment of the molecular properties of the derivatives against BSH by molinspiration calculations for their drug likeness. Imipenem seems to be the potent inhibitory drug to target BSH with a promising binding energy of -5.54 with nine hydrogen bonds. Drug likeness parameters recorded imipenem as a promising protease and enzyme inhibitor followed by other beta lactam derivatives. The findings of the study emphasize that the beta lactam derivatives show promising interaction with bile salt hydrolase of E.faecalis. The findings of the study can thus have promising implications in combating the menace of drug resistance among E.faecalis associated with endodontic and other systemic infections with further in-vivo validation targeting the same.
\end{abstract}

KEY WORDS: BSH, E.FAECALIS, BETA-LACTAMS, AUTO-DOCK, IN-SILICO.

\section{INTRODUCTION}

Enterococcus faecalis, a gram positive diplococci, is an opportunistic pathogen commonly observed in oral

\section{ARTICLE INFORMATION}

*Corresponding Author: smilinejames25@gmail.com

Received 5th June 2020 Accepted after revision 10th August 2020

Print ISSN: 0974-6455 Online ISSN: 2321-4007 CODEN: BBRCBA

Thomson Reuters ISI Web of Science Clarivate Analytics USA and Crossref Indexed Journal

\section{Clarivate
Analytics}

NAAS Journal Score 2020 (4.31) SJIF: 2020 (7.728)

A Society of Science and Nature Publication,

Bhopal India 2020. All rights reserved.

Online Contents Available at: http//www.bbrc.in/

Doi: http://dx.doi.org/10.21786/bbrc/13.7/49 and other systemic diseases. Pertained to endodontic infections, it has been listed as one among the 25 pathogens (Anderson et al., 2016) that can lead into further complications of the root canal. However, the frequency of the dental and oral manifestations varies with individuals based on oral hygiene, age, gender, comorbidities, geographical distribution and other associated risk factors (Sun et al., 2012). They are capable of causing a variety of infections including endocarditis, sepsis, surgical wound infections, and urinary tract infections (Van Tyne et al., 2013). In recent years, the pathogen has emerged as drug resistant forms due to its drug resistant properties against the routine antimicrobials (Sedgley et al., 2005). Amidst many

$$
291
$$


intrinsic and acquired resistance mechanisms, the vital role of bile salt hydrolase (BSH) in inducing tolerance to bile salts, has an altering effect on the action of beta lactam group of drugs.

This is due to the fact that BSH has a unique allosteric catalytic property that can de-conjugate the bile salts and is considered to be evolutionary related to penicillin $\mathrm{V}$ acylase (PVA), which is responsible for hydrolysing penicillin V (Chand et al., 2018). It is industrially used in the hydrolysis of penicillin $\mathrm{V}$ to produce 6-aminopenicillanic acid (6-APA), which is the precursor molecule for semi-synthetic $\beta$-lactam antibiotics (Chandra et al., 2005).In addition, the nonsubstrate ligand of PVA has a marked modulating and hydrolysing ability on BSH (Chand et al., 2016).

The $\beta$-lactam family is the most important class of antibiotics for clinical use as antimicrobial agents and constitute a major part of the global antibiotic market (Rodríguez et al., 2011).Clavulanic acid, thienamycin, cefoxitin, imipenem have shown susceptibility against E. faecalis characterized from various clinical specimens, with varying efficacies. Clavulanate, being considered as a potent inhibitor of $\beta$-lactamase, shows $80 \%$ susceptibility against E.faecalis isolated from urinary tract infections (Venkatesan et al., n.d.). Theinamycin, having a broad spectrum activity to several resistant pathogens, shows synergistic activity with other aminoglycosides against E. faecalisbut lacks in-vivo or in-vitro activity when administered as a single drug (Eliopoulos and Moellering, 1981). Cefoxitin, being active against most bacteria that have become refractory, was not effective against E.faecalis (Moellering et al., 1974). Imipenem are broad-spectrum parenteral antibacterial agents that are generally reserved for use as agents of last resort in the treatment of serious nosocomial infections (Karlowsky et al., 2018).Even the extremely broad spectrum drug imipenem shows resistance among vancomycin resistant E.faecalis (Ono et al., 2005).

BSH being evolutionarily related to penicillin $\mathrm{V}$ hydrolase, it would be a best target to combat the clinical complication of E.faecalis infections and its emerging resistance against the penicillin derivatives in an alternate way. The present investigation is thus aimed to assess the potent inhibitory role of four beta lactam derivatives viz., clavulanic acid, thienamycin, cefoxitin and imipenem against BSH by in-silico docking analysis. Thus will aid in the selection of the best beta lactam derivatives targeting BSH of Enterococcus faecalis to treat endodontic and other systemic infections.

\section{METHODS}

Retrieval And optimisation of BSH: From the RCSB protein data bank (http://www.rcsb.org/pdb) the crystal structure of Bile salt hydrolase was retrieved for this. For the target optimization the hydrogen atoms were added and using kollman united atoms force field, the electronic charges were assigned to the protein using AutoDock
Tool -1.5.6. RASMOL tool was applied to view the three dimensional structure of BSH.

Preparation and optimization of the ligand: Using Chemsketch Software the structures of the beta lactam derivatives viz., clavulanic acid, thienamycin, cefoxitin, and imipenem were drawn and generation of their 3-D structures and 3D optimization was also done. The selected ligands were further saved in .mol file followed subsequent conversion using open babel molecular converter program and were saved in PDB format.

Molinspiration assessments: Molecular descriptors such as logP for partition coefficient, molecular weight of the compounds, the counts of hydrogen bond acceptors and donors relates to the membrane permeability and bio-availability of the compounds were assessed using molinspiration (Rizvi et al., 2013). Characterization of the absorption, distribution, metabolism and elimination (ADME) properties of the selected compounds was checked to fit into the Lipinsky's rule of five (Morris et al., 1998).

Auto-docking for drug-ligand interactions: The docking analysis to interpret the affinity between clavulanic acid, thienamycin, cefoxitin, and imipenem against BSH of E.faecalis was achieved by an auto-dock tool. Briefly, the target protein is embedded in a grid in pre-calculated grid maps (Autodock 4.2) (Ertl et al., 2000). AutoDock requires pre-formed grid maps, one for each atom type present in the ligand being docked. Calculation of the maps was done using the Autogrid auxiliary program by allowing the flexible molecules to move in the six spatial degrees of freedom for orientation and torsion. For the modelling of H-bonds and vanderWaals interactions, we implemented Lennard-Jones parameters 12-10 and 12-6respectively.Evaluation of the force field was done in two steps, first by estimating the intra-molecular energetics between the transition from unbound states to the ligand-protein bound state. Secondly, evaluation of the intermolecular energetics was done by combining the ligand and protein in their bound conformation in the $\Delta \mathrm{G}=\Delta \mathrm{Gvdw}+\Delta \mathrm{Ghbond}+\Delta$ Gelec $+\Delta$ Gtor + $\Delta \mathrm{Gdesolv}$ format. First three terms are for van der Waal's, hydrogen bonding, electrostatics respectively (Lipinski et al., 1997).

Visualisation of the docked molecules: The hydrogen bond interactions clavulanic acid, thienamycin, cefoxitin, and imipenem against BSH of E.faecalis were visualized using Discovery Studio Visualizer. The relative stabilities were evaluated using their molecular dynamics, binding affinities, energy simulations with further docking score assessments.

\section{RESULTS AND DISCUSSION}

Structure retrieval of the BSH protein from E.faecalis: The Crystal structure of a trimeric variant of the Bile Salt Hydrolase from E.faecalis(strain T2) is downloaded from PDB database and its structure is documented as 4WL3-A Chain. Optimization was successfully achieved 
by the removal of the water molecules with merging of hydrogen atoms to the receptor molecule.RASMOL tool showed 3D structure of BSH with pink colour indicating the alpha-helix, yellow arrow indicating the beta sheets and white colour indicating the turns.

Beta lactam derivatives structure optimizations (the ligands): Optimization of the ligand was successful using ACD Chemsketch and the compatible format was retrieved using Open Babel molecular converter tool. The retrieved 2D and 3D structures of the ligands and its SMILES format are shown in table 1.
Drug likeness by molinspiration estimation: The bioactivity scores prediction of clavulanic acid, thienamycin, cefoxitin, and imipenem against BSH of E.faecalis based on the calculation of the GPCR ligand, ion channel modulation, kinase inhibitor, nuclear receptor ligand, protease inhibition and enzyme inhibition towards drug likeness is scored and tabulated in Table 2. Imipenem scores to be the best drug candidate followed by apigenin and cirsimaritin in targeting the bile salt hydrolase.

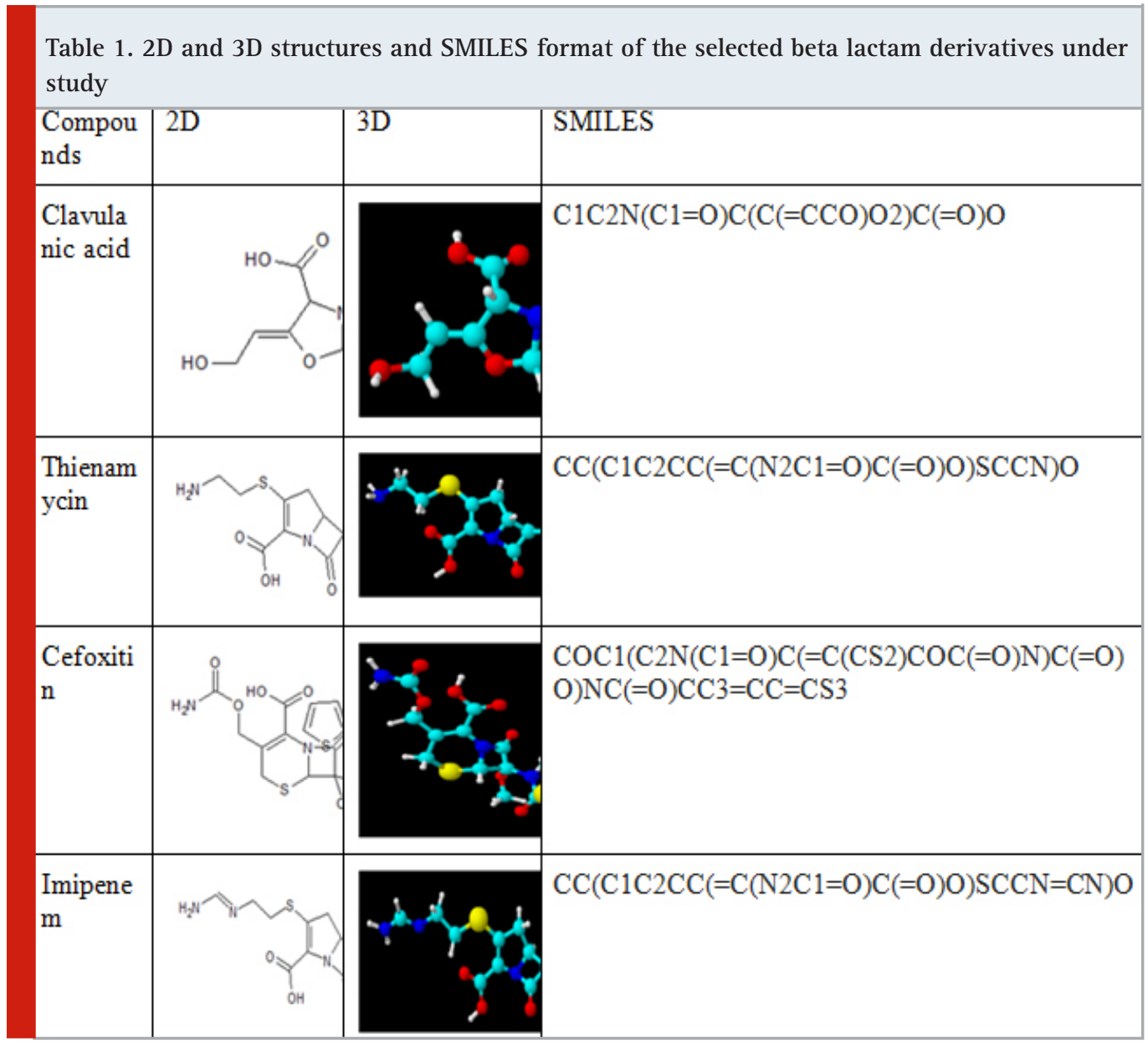

Docking analysis of the beta lactam derivatives against BSH of E.faecalis: Based on the lowest energy and minimal solvent accessibility, selection of the best conformers was made using LGA of the docked structure. Accelrys discovery studio visualization of the hydrogen bond interactions in the stick model between the Clavulanic acid, Thienamycin, Cefoxitin, and Imipenem against BSH of E. faecalis is given in Figure 1,2,3\& 4. The number of hydrogen bonds formed, torsional energy and the docking scores between the drug and ligands are given in Table 3.

The present study involved an in-silico approach to target bile salt hydrolase of E.faecalis, to achieve a preliminary understanding of the drug interactions using computational tools prior experimental approaches. In-sights into such an approach reveals the potent virulent based interactions which will further enhance the application of effective therapeutics in endodontic treatments (J et al., 2018). Structure based computational analysis in drug designing with the 3D knowledge in the prediction of the receptor bound to the ligands with a given or congeneric molecule optimization by molecular docking has spurred interest in recent decades in the field of dentistry. The conformational flexibility of virulent protein playing a vital role in endodontic infections is best achieved by docking analysis which is otherwise a perplexing problem and quite challenging.

In the present study, the beta lactam derivatives viz., clavulanic acid, thienamycin, cefoxitin and imipenem docked against the bile salt hydrolase of E.faecalis resulted in a promising receptor - ligand complex. Computational docking analysis predicts the exact 
orientation of the protein-ligand docked structures based on the scores achieved by pose and strength (Wang et al., 2003). In addition, the molecular surface structures and interaction of BSH of E. faecalis with the beta lactam drugs were analysed based on several properties like interactive charges, and other chemical bonds like Vander Waal's forces which is further determined by hydrogen bonding (Jiang and Kim, 1991). In this context, accelrys studio visualised and docked structures of BSH of E.faecalis yielded excellent docking scores with promising hydrogen bond interactions and the affinity distance scores for the beta lactam derivatives and $\mathrm{BSH}$ interactions.

Table 1. 2D and 3D structures and SMILES format of the selected beta lactam derivatives under study

\begin{tabular}{|l|c|c|c|c|c|c|c|c|c|c|}
\hline Compounds & M. wt & $\begin{array}{c}\text { Mol } \\
\text { formula }\end{array}$ & $\begin{array}{c}\mathrm{H} \text { Bond } \\
\text { Donor }\end{array}$ & $\begin{array}{c}\mathrm{H} \text { Bond } \\
\text { Acceptor }\end{array}$ & Log P & TPSA & $\begin{array}{c}\text { Rotatable } \\
\text { bonds }\end{array}$ & Vol. & N \\
atoms & Compounds \\
\hline Clavulanic acid & 199.162 & $\mathrm{C}_{8} \mathrm{H}_{9} \mathrm{NO}_{5}$ & 2 & 6 & -2.42 & 2 & 0 & 87.07 & 161.4 & 14 \\
\hline Thienamycin & 272.319 & $\mathrm{C}_{11} \mathrm{H}_{16} \mathrm{~N}_{2} \mathrm{O}_{4} \mathrm{~S}$ & 4 & 6 & -1.32 & 5 & 0 & 103.86 & 232.02 & 18 \\
\hline Cefoxitin & 427.446 & $\mathrm{C}_{16} \mathrm{H}_{17} \mathrm{~N}_{3} \mathrm{O}_{7} \mathrm{~S}_{2}$ & 4 & 10 & 0.02 & 8 & 0 & 148.27 & 337.31 & 28 \\
\hline Imipenem & 299.345 & $\mathrm{C}_{12} \mathrm{H}_{17} \mathrm{~N}_{3} \mathrm{O}_{4} \mathrm{~S}$ & 4 & 7 & -0.86 & 6 & 0 & 116.222 & 255.28 & 20 \\
\hline
\end{tabular}

Drug likeness calculation of beta lactam derivatives against BSH of E.faecalis Table 2. Molinspiration calculations of beta lactam derivatives

\begin{tabular}{|l|c|c|c|c|c|c|}
\hline Compounds & $\begin{array}{c}\text { GPCR } \\
\text { ligand }\end{array}$ & $\begin{array}{c}\text { Ion } \\
\text { channel } \\
\text { modulator }\end{array}$ & $\begin{array}{c}\text { Kinase } \\
\text { inhibitor }\end{array}$ & $\begin{array}{c}\text { Nuclear } \\
\text { receptor } \\
\text { ligand }\end{array}$ & $\begin{array}{c}\text { Protease } \\
\text { inhibitor }\end{array}$ & $\begin{array}{c}\text { Enzyme } \\
\text { inhibitor }\end{array}$ \\
\hline Clavulanic acid & -0.34 & -0.02 & -0.98 & -0.43 & 0.70 & 0.80 \\
\hline Thienamycin & 0.09 & -0.19 & -0.60 & -0.38 & 1.00 & 0.89 \\
\hline Cefoxitin & -0.33 & -0.49 & -0.82 & -0.64 & 0.70 & 0.01 \\
\hline Imipenem & 0.29 & -0.22 & -0.58 & -0.24 & 1.15 & 0.98 \\
\hline
\end{tabular}

Table 3. Overall docking results of ligands with BSH of E.faecalis

\begin{tabular}{|l|c|c|c|c|c|c|c|c|}
\hline $\begin{array}{l}\text { BSH docking } \\
\text { with compounds }\end{array}$ & $\begin{array}{c}\text { Number of } \\
\text { hydrogen } \\
\text { bonds }\end{array}$ & $\begin{array}{c}\text { Binding } \\
\text { energy }\end{array}$ & $\begin{array}{c}\text { Ligand } \\
\text { efficiency }\end{array}$ & $\begin{array}{c}\text { Intermolecular } \\
\text { energy } \\
\text { desolv } \\
\text { Energy }\end{array}$ & $\begin{array}{c}\text { vdW + H } \\
\text { Bond + }\end{array}$ & $\begin{array}{c}\text { Electrostatic } \\
\text { energy }\end{array}$ & $\begin{array}{c}\text { Torsional } \\
\text { energy } \\
\text { Unbound }\end{array}$ \\
$\begin{array}{c}\text { Total } \\
\text { internal }\end{array}$ \\
\hline Clavulanic acid & 6 & -5.4 & -0.39 & -6.59 & -6.59 & 0.01 & 1.19 & -1.29 \\
\hline Thienamycin & 6 & -4.24 & -0.24 & -6.63 & -5.54 & -1.09 & 2.39 & -3.73 \\
\hline Cefoxitin & 5 & -4.63 & -0.17 & -7.32 & -7.22 & -0.1 & 2.68 & -3.22 \\
\hline Imipenem & 9 & -5.54 & -0.28 & -8.23 & -7.36 & -0.86 & 2.68 & -1.51 \\
\hline
\end{tabular}

Figure 1: Accelrys discovery studio visualisation of the hydrogen interactions between BSH and clavulanic acid

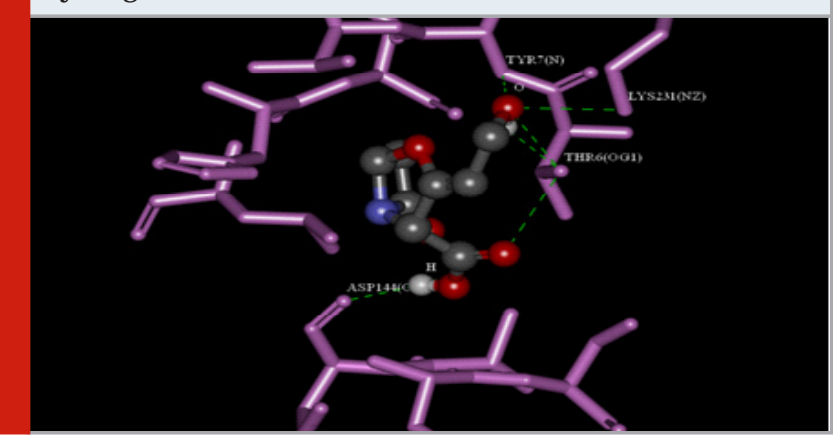

Figure 2: Accelrys discovery studio visualisation of the hydrogen interactions between BSH and thienamycin

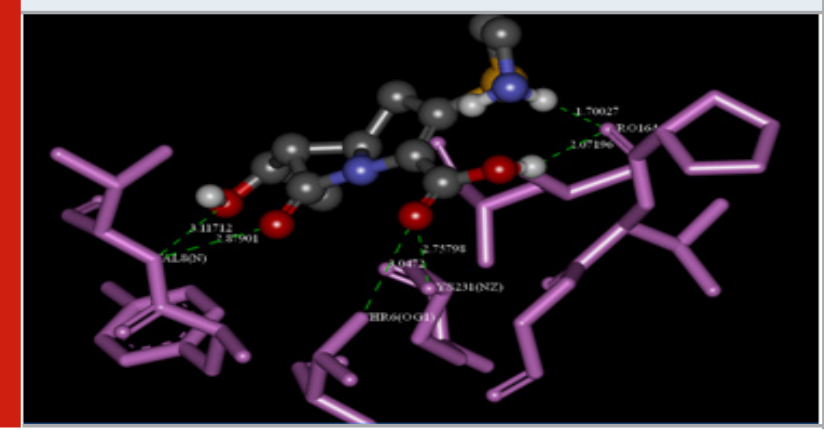


The number of hydrogen bonds between the donors and the acceptors determines the best conformers and the binding energy values predicts the bioactivity value for a ligand to the corresponding receptor suggesting the inhibitory effect of the drugs to target E. faecalis based endodontic infections mediated by BSH. In this view, imipenem scores to be the best inhibitory drug of choice against BSH with nine hydrogen bonds followed by clavulanate and thienamycin with six bonds and finally by cefoxitin with five hydrogen bonds. The promising inhibitory activity of imipenem has already been documented in an earlier study (Beadle and Shoichet, 2002) and in the present investigation, we suggest BSH of E. faecaliscan be best targeted by imipenem.

Figure 3: Accelrys discovery studio visualisation of the hydrogen interactions between BSH and cefoxitin

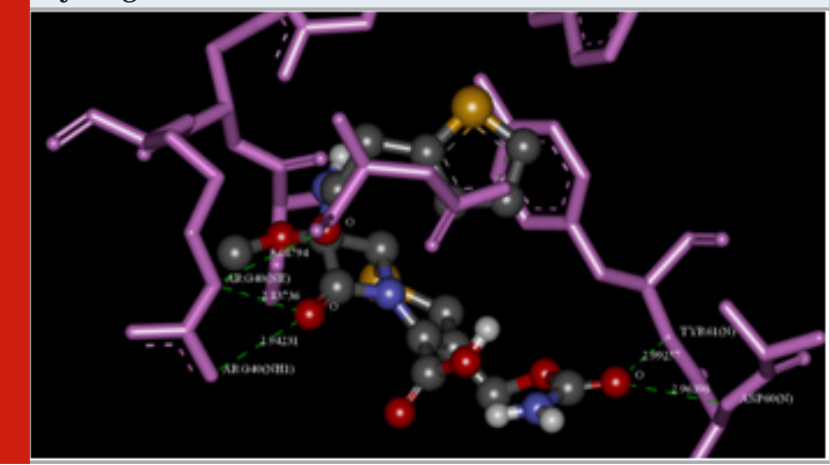

Figure 4: Accelrys discovery studio visualisation of the hydrogen interactions between BSH and imipenem

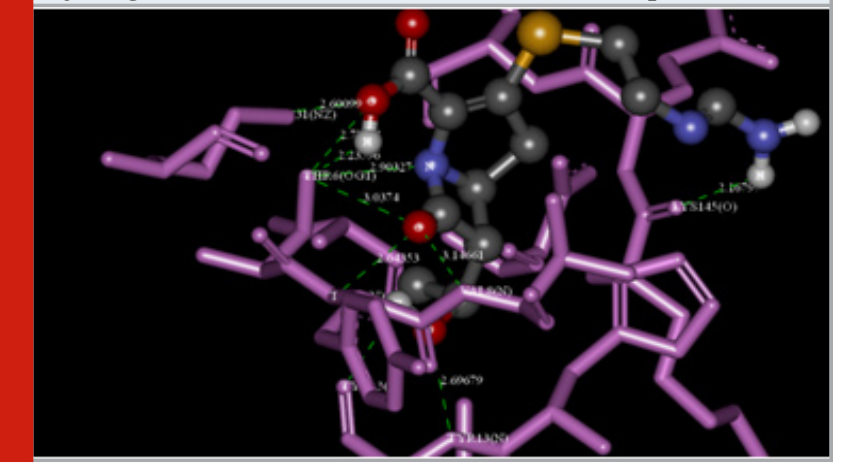

We used auto dock 4.2 to evaluate the interactions of E.faecalis BSH, which is considered as a suite of automated docking tools with a software for modelling flexible small molecule binding to 3D structures of receptor proteins. Using genetic algorithms for the conformational search a rapid grid based method of energy evaluationofE. faecalis BSH with the drugs was achieved in the study in analysing the docking simulations. In this study, the Lamarckian Genetic Algorithm (LGA) was used to explore the binding conformational landscape of the beta lactam derivatives chosen for inhibiting the BSH. The docking scores on bile salt hydrolase indicated that there is a direct relationship between the energy of the binding affınity, referring to the lowest docking scores and the stability. In accordance with this, the binding scores between the beta lactam derivatives and BSH in the present study showed potent binding affinity to the binding sites with $-5.4,-4.24,-4.63$ and $-5.54 \mathrm{Kcal} / \mathrm{mol}$ for clavulanic acid, thienamycin, cefoxitin and imipenem respectively with more potent and stable binding towards imipenem with the active sites of BSH. In addition, the intermolecular energy, van der waals energy and torsional energy were also at a higher end for imipenem when compared to the other three beta lactam derivatives and the beta lactam inhibitor clavulanate in interacting with the BSH of E.faecalis.

In the present study, molinspirational results were very promising with the n-violations of the beta lactam derivatives against BSH as zero satisfying the Lipinsky's rule of five. The application of molinspiration calculations of beta lactam derivatives with bile salt hydrolase suggests its importance in the practical approach to calculate the molecular properties and predicted the bioactivity scores to assess the best inhibitory agent for BSH. In molinspiration analysis, topological polar surface area (TPSA) of a molecule is considered as a useful descriptor to characterize the drug absorption and bioavailability and the values of TPSA and $\mathrm{OH}-\mathrm{NH}$ interactions indicate that the selected ligands viz, the beta lactam derivatives to possess a smooth and efficient binding to the target proteins. It is a known fact that the drug molecules with TPSA values of $>140 \AA$ or higher possess a low-absorption (miLogP) substantiating its role for its oral bioavailability for the drugs (Davies et al., 2007), (Priyadharsini et al., 2018). In this context, except cefoxitin all the other ligands score high absorption with clavulanate with high membrane penetration with a TPSA score of 87.07.

In the present study, we also predicted the bioactivity scores of clavulanic acid, thienamycin, cefoxitin, and imipenem towards drug likeness by calculating the activity scores of various inhibitor, ligand and modulator effects. All the derivatives scored above 0.20 as potent protease inhibitor and enzyme inhibitor except cefoxitin (Table $3 b$ ) against BSH of E.faecalis.

\section{CONCLUSION}

This work represents an advance in biomedical sciences in dentistry as there is a high possibility of orienting and scoring of the binding site of any target protein by molecular docking, which is a promising computer based drug design tool. The present investigation thus targets the BSHofE.faecalis, which is a potent virulent and pathogenic mechanism portraying challenge in the treatment strategies in E.faecalis associated endodontic diseases. In addition, BSH being an evolutionary analogue to penicillin $\mathrm{V}$ hydrolase, assessment of the best inhibitory beta lactam derivatives through molecular docking is promising to determine the potent drug of choice to combat resistant forms of E.faecalis in an alternate way by inhibiting the bile salt hydrolase. The present study is concluded by stating imipenem as the best inhibitory agent in targeting the BSH of E.faecalis towards arresting the bile resistance however requiring further in-vitro experimental validation. 


\section{ACKNOWLEDGEMENTS}

The authors would like to acknowledge the help and support rendered by the Department of Microbiology of Saveetha Dental College and Hospitals and the management for their constant assistance with the research.

Conflict of Interest: None to declare

\section{REFERENCES}

Anderson, A.C., Jonas, D., Huber, I., Karygianni, L., Wölber, J., Hellwig, E., Arweiler, N., Vach, K., Wittmer, A., Al-Ahmad, A., 2016. Enterococcus faecalis from Food, Clinical Specimens, and Oral Sites: Prevalence of Virulence Factors in Association with Biofilm Formation. Frontiers in Microbiology.

Beadle, B.M., Shoichet, B.K., 2002. Structural Basis for Imipenem Inhibition of Class $C \beta$-Lactamases. Antimicrobial Agents and Chemotherapy.

Chand, D., Panigrahi, P., Varshney, N., Ramasamy, S., Suresh, C.G., 2018. Structure and function of a highly active Bile Salt Hydrolase (BSH) from Enterococcus faecalis and post-translational processing of BSH enzymes. Biochim. Biophys. Acta: Proteins Proteomics 1866, 507-518.

Chand, D., Ramasamy, S., Suresh, C.G., 2016. A highly active bile salt hydrolase from Enterococcus faecalis shows positive cooperative kinetics. Process Biochemistry.

Chandra, P.M., Manish Chandra, P., Brannigan, J.A., Prabhune, A., Pundle, A., Turkenburg, J.P., Guy Dodson, G., Suresh, C.G., 2005. Cloning, preparation and preliminary crystallographic studies of penicillin $\mathrm{V}$ acylase autoproteolytic processing mutants. Acta Crystallographica Section F Structural Biology and Crystallization Communications.

Davies, T.A., Page, M.G.P., Shang, W., Andrew, T., Kania, M., Bush, K., 2007. Binding of Ceftobiprole and Comparators to the Penicillin-Binding Proteins of Escherichia coli, Pseudomonas aeruginosa, Staphylococcus aureus, and Streptococcus pneumoniae. Antimicrobial Agents and Chemotherapy.

Eliopoulos, G.M., Moellering, R.C., 1981. Susceptibility of enterococci and Listeria monocytogenes to $\mathrm{N}$-Formimidoyl thienamycin alone and in combination with an aminoglycoside. Antimicrobial Agents and Chemotherapy.

Ertl, P., Rohde, B., Selzer, P., 2000. Fast calculation of molecular polar surface area as a sum of fragment-based contributions and its application to the prediction of drug transport properties. J. Med. Chem. 43, 37143717.

Jiang, F., Kim, S.H., 1991. "Soft docking”: matching of molecular surface cubes. J. Mol. Biol. 219, 79-102.

J, V.P., SmilineGirija, A.S., Paramasivam, A., 2018. Enterococcus faecalis an Emerging Microbial Menace in Dentistry-An Insight into the In-Silico Detection of Drug Resistant Genes and Its Protein Diversity. JOURNAL OF

\section{CLINICAL AND DIAGNOSTIC RESEARCH.}

Karlowsky, J.A., Lob, S.H., Kazmierczak, K.M., Young, K., Motyl, M.R., Sahm, D.F., 2018. Activity of ImipenemRelebactam against Clinical Isolates of Gram-Negative Bacilli Isolated in Hospital Laboratories in the United States as Part of the SMART 2016 Program. Antimicrob. Agents Chemother. 62.

Lipinski, C.A., Lombardo, F., Dominy, B.W., Feeney, P.J., 1997. Experimental and computational approaches to estimate solubility and permeability in drug discovery and development settings. Advanced Drug Delivery Reviews.

Moellering, R.C., Dray, M., Kunz, L.J., 1974. Susceptibility of Clinical Isolates of Bacteria to Cefoxitin and Cephalothin. Antimicrobial Agents and Chemotherapy.

Morris, G.M., Goodsell, D.S., Halliday, R.S., Huey, R., Hart, W.E., Belew, R.K., Olson, A.J., 1998. Automated docking using a Lamarckian genetic algorithm and an empirical binding free energy function. Journal of Computational Chemistry.

Ono, S., Muratani, T., Matsumoto, T., 2005. Mechanisms of Resistance to Imipenem and Ampicillin in Enterococcus faecalis. Antimicrobial Agents and Chemotherapy.

Priyadharsini, J.V., Vijayashree Priyadharsini, J., Smiline Girija, A.S., Paramasivam, A., 2018. An insight into the emergence of Acinetobacter baumannii as an oro-dental pathogen and its drug resistance gene profile - An in silico approach. Heliyon.

Rizvi, S.M.D., Shakil, S., Haneef, M., 2013. A simple click by click protocol to perform docking: AutoDock 4.2 made easy for non-bioinformaticians. EXCLI J. 12, 831-857.

Rodríguez, M., Núñez, L.E., Braña, A.F., Méndez, C., Salas, J.A., Blanco, G., 2011. Mutational Analysis of the Thienamycin Biosynthetic Gene Cluster fromStreptomyces cattleya. Antimicrobial Agents and Chemotherapy.

Sedgley, C.M., Molander, A., Flannagan, S.E., Nagel, A.C., Appelbe, O.K., Clewell, D.B., Dahlén, G., 2005. Virulence, phenotype and genotype characteristics of endodontic Enterococcus spp. Oral Microbiol. Immunol. 20, 10-19.

Sun, J., Sundsfjord, A., Song, X., 2012. Enterococcus faecalis from patients with chronic periodontitis: virulence and antimicrobial resistance traits and determinants. European Journal of Clinical Microbiology A Infectious Diseases.

Van Tyne, D., Martin, M., Gilmore, M., 2013. Structure, Function, and Biology of the Enterococcus faecalis Cytolysin. Toxins.

Venkatesan, K.D., Chander, S., Ananthi, B., Abigail, S., Victor, K., n.d. Antibiotic resistance pattern of Enterococcal isolates from patients with urinary tract infection.

Wang, R., Lu, Y., Wang, S., 2003. Comparative Evaluation of 11 Scoring Functions for Molecular Docking. Journal of Medicinal Chemistry. 